\title{
The Higgs Quantum Space Dynamics Generating the Gravitational Dynamics in the Universe
}

\author{
Jacob Schaf
}

Institute of Physics, Federal University of Rio Grande do Sul, Porto Alegre-RS, Brazil

Copyright (C)2015 Horizon Research Publishing All rights reserved.

\begin{abstract}
The present work makes use of the recent experimental observations, achieved with the help of the tightly synchronized clocks in orbit, together with recent theoretical achievements of the Higgs theory to understand the gravitational physics. According to the Higgs theory, a Higgs quantum condensate, present throughout space, gives mass to the elementary particles by the Higgs mechanism. Hence, this Higgs Quantum Space (HQS) rules the inertial motion of the elementary particles and is locally the ultimate reference for rest and for motions. Many recent experimental observations demonstrate that this HQS is moving round earth and round the sun according to Keplerian velocity fields, consistent with the local main astronomical motions. Inertial motion of matter and propagation of light within this Keplerian velocity field is the quintessence of the gravitational physics. In the velocity field of the sun, the planets are locally very closely stationary with respect to the moving HQS. This explains the null results of the Michelson light anisotropy experiments. It also explains the absence of the gravitational time dilation, due to the solar gravitational potential, on the GPS clocks, which is predicted by General Relativity, but not observed. Inertial motion of bodies within the earth's Keplerian velocity field gives origin to the gravitational acceleration on earth. In the current theories, space itself is idle and plays no active role in the gravitational dynamics. These theories ascribe the whole dynamics to interaction forces, which leads to the actual persistent troubles with gravitation.
\end{abstract}

Keywords Gravity, Gravitational Physics, Gravitational Fields, Gravitational Effects, Higgs Condensate, Higgs Quantum Space

\section{Introdution.}

Although using concepts of the Higgs theory, the aim of this work, is not elementary particle physics, but the gravitational physics. The development of this work is wholly made in terms of the interpretation of experimental observations. The gravitational dynamics will be seen as result of inertial motion (wave propagation) of the particles and light in a warping Higgs Quantum Space (HQS), ruling the iner- tial motion of matter and propagating light. Therefore, it is important to recall some relevant properties of wave propagation. The propagation of waves, as a rule, is along the normal to the wave fronts. Therefore, if the real HQS is moving non-uniformly, then the wave fronts will be rotated according to a time rate and propagate along a curve. Electromagnetic waves and the wave functions of quantum mechanics propagate in the vacuum. Several experiments (Michelson), searching for evidence of a medium of propagation in the vacuum, failed. In Section IV of this article, it will be shown why they failed and that this failure is intimately connected with the physical mechanism of gravity.

According to the Theory of Relativity, empty space (vacuum) contains nothing that can represent a reference for motions. Accordingly, it is usually assumed that these waves need no medium of propagation. Many physicists assert that the wave-functions $\left(\psi(r, t)=\psi(r) e^{i(k r-w t)}\right)$ of quantum mechanics, where $\psi(r)$ is an amplitude, $k=2 \pi / \lambda$ is the wave vector, $\lambda$ is the wavelength, $w$ is the angular frequency and $w / k$ is the velocity of propagation, are purely mathematical constructions that have no tangible reality. Only the product $\psi^{*} \psi=\psi^{2}(r)$, in which the imaginary part is absent, is an observable. The most accepted interpretation is that $\psi^{*} \psi$ describes the probability density, the probability of observing the particle within the small volume of a detector. However, in this probabilistic interpretation, the position of the associated hypothetical particle has an odd intrinsic arbitrariness that is explained in terms of the principle of uncertainty.

In fact many experimental observations show that the wave-functions (wave-packets) of particles (de Broglie waves) and of light (electromagnetic waves) have fundamental differences. For instance, in the two-slit interference experiment with individual photons, the amplitude of the wave function normally has many maxima and minima on the screen. Observation nevertheless shows that the photon hits the screen at only one unique point. The way the photon chooses this point seems to be a true game of dice. However, after shooting many individual monochromatic photons through the two-slit device, the hits on the screen distribute them according to an interference figure, consistent with the amplitude distribution of the wave function. This shows that this game of dice follows well-defined rules. Any attempt to find out through which slit the photon has passed, results in destruction of the interference figure. This demonstrates that the photon really behaves as a wave until hitting the screen. 
In an analogous two-slit experiment with electrons the electron wave functions follow entirely analogous interference rules as those of photons. However, applying an electric or magnetic field on the two-slit reveals a radical difference. While the photon interference figure remains unaltered, that of the electrons changes completely. Even if the magnetic field $\vec{B}$ falls to zero on the slits ( $\operatorname{curl} \vec{A}=0)$, but an irrotational vector potential $\vec{A} \neq 0$ with different directions or differing intensities is present on the slits, the interference fringes are displaced, as shown by the Aharonov-Bohm experiment. [1] These observations show that the nature of the de Broglie wave-functions of the electrons are fundamentally different from those of the photons. The difference is electric charge. Electric charge allows the electron wave functions to couple to electric, magnetic and even to irrotational vector potential fields. Many experimental observations have evidenced other fundamental differences between the photon and the electron wave functions, the most relevant will be discussed hereafter.

The de Broglie wave functions of particles can differ from the photon wave functions by a series of intrinsic parameters, like rest mass, spin, electric charge, hypercharges, isospin etc. Note however, that all these parameters are intrinsically quantized, which evidences that they are essentially wave mechanic features. Quantization is an exclusive feature of wave mechanisms. Quantization of particle states, confined in potential wells, is well understood in terms of conventional quantum mechanics. The intrinsic quantization too is due to spatial confinement. However, in the intrinsic case, confinement has a completely different origin, related with energy minimization. Intrinsic quantization is an inherent feature of all excitations in quantum fluids (superfluids and superconducting condensates).

It is clear that, although the conventional quantum mechanics description of the motion of particles in terms of wave functions explains several behavioral features of light and of particles, it is far from a complete explanation. Why do the wave-packets of light (gauge particles) propagate in the vacuum always at the fixed velocity $c=1 / \sqrt{\mu_{0} \times \epsilon_{0}}$, where $\mu_{0}$ and $\epsilon_{0}$ are respectively the permeability and the permittivity of the vacuum, while the wave-packets of particles move in the same vacuum at any velocity lower than $c$ and never $c$ ? Why do de Broglie wave functions of particles resist changes of velocity? Why do particles obey the principle of inertia? The usual answer to these questions is that particles have rest mass (relativistic mass), while photons have not. This explanation however is shorthand, because it does not explain why the wave-packets of particles have an associated rest mass.

In the gauge theories, all the elementary particles are originally mass-less and move at the velocity of light. However, opposing the gauge theories, experimental observations have shown that the weak nuclear interaction is short-range and that the gauge bosons $\left(W^{+}, W^{-}\right.$and $\left.Z\right)$, mediating it, have large masses. Deciphering the origin of the inertial mass of these gauge particles was one of the most challenging puzzles of physics. It took decades of intense efforts and involved dozens of brilliant scientists.

The clue to solve this mystery came from superconductivity. Superconductors confine the magnetic field by the Meissner effect in the form of quantized magnetic fluxons that are screened from the superconducting condensate (SCC) by quantized Abrikosov vortices. Abrikosov vortices are solenoidal circulation fields of the charged SCC round the quantized magnetic fluxons (screening currents), induced by the solenoidal vector potential $\vec{A}$, associated with the magnetic fluxons. Confinement of the electromagnetic field makes it short-range and gives inertial (rest) mass to the photons within superconductors. [2] This momentous achievement was a major breakthrough and provided an important hint in the search for the origin of the inertial mass of the elementary particles. [3] It suggested that a quantum condensate, analogous to the SCC, must be present throughout space, confining the weak and strong nuclear fields, making them short-range and thereby giving mass to the elementary particles.

A series of ground breaking achievements [4, 5, 6, 7, 8], which will not be discussed here, ended in the Higgs mechanism, responsible for the origin of mass of the elementary particles. The Higgs mechanism, confining the weak nuclear field, is the Higgs Quantum Space (HQS) analog of the Meissner effect in superconductivity. The Higgs theory of the Standard Elementary Particle Model (SEPM) introduces profound changes in the current view about the nature of space and of the motions. The presence of the real Higgs condensate (HC) throughout space puts in check the idea of the Special Theory of Relativity (STR), according to which space in itself contains nothing real that can represent a reference for motions. The HQS is not only a local ultimate reference for the motions of particles and light, however literally rules these motions. The law of inertia turns out to be simply a modified law of propagation.

In concluding this introduction, it is important to underline that endless experimental observations demonstrate that motion of the elementary particles and light effectively obeys the rules of wave propagation. In the new scenario of the HQS, the velocity of light has locally a well-defined velocity $c_{H Q S}$ with respect to this HQS and not with respect to Einstein's inertial references. Recent experimental observations, $[9,10,11]$ and their interpretation $[12,13,14]$ demonstrate that the HQS is itself moving round earth and round the sun etc., according to Keplerian velocity fields, consistent with the local main astronomical motions. Visibly, the astronomical bodies throughout the universe follow very closely the motion of the HQS (circular equatorial orbits) in the respective Keplerian velocity fields. This is the true reason why the velocity of light is isotropic with respect to earth. This is also the reason why the planetary satellite systems, the solar system as well as the galaxies are disk shaped. Free bodies that do not follow the motion of the HQS cannot have circular nor equatorial orbits. They move along elliptic or non-equatorial orbits. However, these motions are ruled by the principle of inertia. In the extreme elliptic case, the motions correspond to vertical free-fall.

In Sections II and III, the most relevant properties and phenomenologies of respectively the usual quantum condensates and of the Higgs Quantum Space will be discussed. In Sections IV and V the troubles with respectively the Special and the General Theories of Relativity will be highlighted. In Section VI it will be shown that motion of the HQS according to a Keplerian velocity field round the astronomical bodies, consistent with the local main astronomical motions, straightforwardly and appropriately generates, in terms of the rules of wave propagation (inertial motion), the observed gravitational dynamics on earth and in the solar system etc. Section VII describes a large number of experimental observations, 
showing that this HQS-dynamics appropriately creates all the observed effects of the gravitational fields on the propagation of light and on the rate of the clocks.

\section{Quantum Condensates and Their Intriguing Phenomenologies.}

Quantum condensates or Bose-Einstein (BE) condensates are boson systems in a macroscopic quantum ground state. BE condensation takes place because of the quantum phase correlation between the wave functions of the bosons. In sufficiently low temperatures, the frequency of decoherent scatterings reduces, the particle wave-packets become spatially extended and overlapping becomes important. In these conditions, BE phase correlation eventually overcomes the thermal energy and condensation can begin. The boson system can lower its energy by spontaneously breaking the $U(1)$ gauge symmetry and undergoing long-range phase coherence. In this transition all the particles assume the same phase $\left(\theta_{0}\right.$ say), condensing into a same macroscopic quantum phase coherent state. In the condensate, the particles become completely entangled and indistinguishable. Lowering energy by phase coherence is a universal quantum mechanics phenomenon that also occurs between fermions, like for instance in the covalent chemical bond. However, in the fermions case the quantum state cannot become macroscopic because of the Pauli Exclusion Principle.

The charged SCC is described by a complex (two component) macroscopic (Ginsburg-Landau) order parameter $\phi=$ $\phi(x, y, z) e^{i \theta_{0}}$ that represents the resting condition (ground state) of the condensate. In this expression $\phi(x, y, z)$ is the amplitude as a function of position, $\theta_{0}$ is the phase and $\rho(x, y, z)=\phi^{*} \phi$ is the local volumetric condensate density that intrinsically distributes uniformly throughout the volume of the condensate. The complex order parameter allows the condensate to undergo intrinsic (internal) motion without any perceptible classical displacement.

$\mathrm{BE}$ correlation gives rise to a negative potential energy (bonding) term, the value of which increases linearly with the condensate density $\rho=\phi^{*} \phi$. Another positive potential energy (anti-bonding) term arises from repulsive core interaction between the bosons, that increases with the squared density $\left(\phi^{*} \phi\right)^{2}$ and prevents collapse of the system. The effective potential is:

$$
U(\rho)=-n\left(\phi^{*} \phi\right)+m\left(\phi^{*} \phi\right)^{2}
$$

where however the negative coefficient $(-n)$ of the bonding term is considerably larger than the positive coefficient $(m)$ of the anti-bonding term. Therefore the minimum of the effective potential energy occurs not for $\phi^{*} \phi=0$, as would be usual, however for a finite value $\phi^{*} \phi=n / m$. This is known as a non-zero vacuum-expectation-value homogeneous throughout the volume of the condensate.

The deepness of the potential well depends on the strength of the BE correlation. As the phase $\left(\theta_{0}\right)$ can take values within zero and $2 \pi$, the potential energy well is symmetric about $\phi=0$ and thus has the form of a Mexican sombrero in the complex $\phi_{R e}$ and $\phi_{I m}$ plane ( please see Fig.1). While the deepness in the case of the SCC is in the order of $1 \mathrm{meV}$, in the case of the Higgs condensate the deepness is in the order of hundreds of $\mathrm{GeV}$.

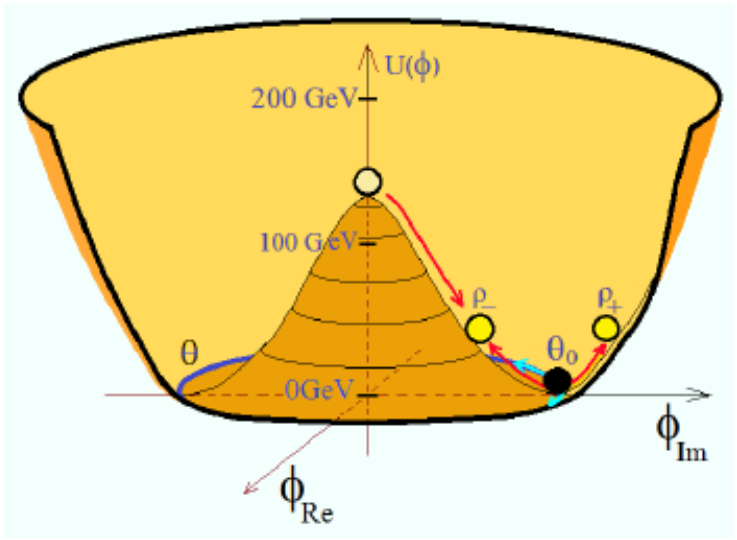

Figure 1. Potential Well of Bose-Einstein Condensates : The figure depicts the form of the Mexican sombrero potential, characteristic of Bose-Einstein condensates (Higgs condensate). The energy scale is per condensed Higgs boson. A red arrow indicates the transition toward the lower energy phase coherent state with the well-defined phase $\theta_{0}$. The figure also indicates the high volumetric density $\left(\rho_{+}\right)$ and low volumetric density $\left(\rho_{-}\right)$states. While $\rho_{+}$drives accelerated expansion, $\rho_{-}$drives accelerated contraction of the condensate. This is the Higgs mode. The Goldstone mode is indicated along the blue bottom circle.

Any local displacement of the phase within a small volume of the condensate, with respect to the overall phase of the order parameter, costs energy, because it must conquer with the local phase correlation and climb up in the potential energy. This gives rise to a peculiar transient quantum phase stiffness that nevertheless manifests it only during new displacement of the phase and enables the quantum condensates to transmit longitudinal as well as transverse elastic oscillations analogously as elastic solids.

Phase displacement of the order parameter of a quantum condensate inherently is associated with flow of the condensate. While a constant phase gradient $(\nabla \theta)$ makes the condensate to flow uniformly without any resistance along the phase gradient with velocity proportional to the magnitude of the gradient, a phase gradient changing with time corresponds to accelerated motion of the condensate. Due to the phase stiffness, such motions, once excited, automatically become persistent or propagate at a characteristic velocity (the velocity of light in the case of the $\mathrm{HC}$ ). For instance, if a current is excited in the superconducting coils of a superconducting magnet by an applied electromotive force, it will flow forever and can be stopped only by an opposite electromotive force.

$\mathrm{BE}$ condensates couple only to specific fields. It is well known that the SCC interacts only with electromagnetic (EM) fields. Superconductivity and magnetic fields are intrinsically incompatible with each other because the vector potential, associated with the magnetic field, causes local phase displacements introducing phase disorder and thereby increasing the energy of the system, locally destroying superconductivity and recovering the $U(1)$ gauge symmetry. Superconductors can lower their energy by developing a macroscopic screening velocity field of the SCC, confining or expelling out the magnetic field. This is the macroscopic effect [15], responsible for the levitation of magnets by superconductors.

In superconductors, stationary circulation fields of the condensate along closed loops (local phase or local Goldstone 
modes) are caused by the vector potential field. A unit of the quantized magnetic flux $\Phi_{o}$ is confined within vortex flow of the SCC along a closed loop. This flow has a locked-in phase displacement $\theta$ along the loop:

$$
\theta=\frac{2}{\pi} \Phi_{o} \oint \vec{A} \cdot d \vec{l}=n 2 \pi
$$

In this equation $\vec{A}$ is the solenoidal vector potential and $d \vec{l}$ is an infinitesimal vector line element along the closed integration path. Single valuedness requires that the total phase displacements $\theta$ round the loops be an integer multiple of $2 \pi$. Equation (2) rules the intrinsic quantization of excitations in quantum fluids. This intrinsic quantization too is due to spatial confinement; however, the origin of this confinement is completely different from that of a usual particle confined by a potential well. Visibly this confinement is ruled by minimization of energy. Displacing the phase over a larger volume of the condensate costs more energy than over a smaller one. The very stable Rotons, Maxons and Vortices in superfluids and Abrikosov vortices in superconductors are examples of such intrinsically quantized quasi-particles.

\section{The Higgs Condensate as a Quan- tum Space, Ruling the Inertial Mo- tion of Matter and the Propagation of Light.}

According to the Standard Elementary Particle Model (SEPM), shortly after the Big-Bang, on cooling through $10^{15}$ degrees Kelvin a scalar Higgs field analogous to the BE correlation and present throughout space, caused spontaneous breaking of the $S U(2) \times U(1)$ doublet of the electroweak symmetry. The broken symmetry resulted in the Higgs condensate (HC) and the photon. Analogously as the SCC, the $\mathrm{HC}$ is described by a complex order parameter, however having two charged and two uncharged components. The two spin 1 components with opposite charges together with the uncharged component with spin 1 then have confined the weak field, giving mass to the $W^{+}, W^{-}$and $Z$ vector bosons by the Higgs mechanism.[6, 7, 8] Moreover, Yukawa like coupling of quarks and leptons (porting hypercharges) to the surviving uncharged spin zero component gives them mass too. Giving mass to these particles, means giving them mechanical properties. This lets clear that the Higgs Quantum Space (HQS) effectively rules the inertial motion of matter and therefore is the locally ultimate reference for rest and for motions.

The Higgs theory gives by the first time a scientifically acceptable explanation to the fact that elementary particles have inertial mass and propagate at any velocity lower than $c$. It discloses the origin of the inertial mass (inertial behavior) of the elementary particles. Confinement of specific fields by specific quantum condensates is the key mechanism.

Both the HC and of the SCC are quantum fluids, which is the main reason why their characteristic phenomenologies are very similar. To each effect of the SCC there is an analogous effect of the HC or Higgs Quantum Space (HQS). For instance, the Higgs mechanism in the HQS is the analog of the Meissner effect in superconductivity. However, while the SCC couples only to electromagnetic (EM) fields, the HQS couples only to the weak nuclear field. It however couples indirectly to all particles porting hypercharges (quarks and leptons) by a Yukawa-like mechanism. A superconductor (SC) reacts to an applied EM field in the sense of canceling it within the SC. The HQS analogously confines the nuclear fields, canceling them within space (HQS). While the vector potential of a constant magnetic field causes a constant phase gradient $(\nabla \theta)$, putting the nearby SCC into motion according to a certain velocity field (screening currents), a weak nuclear field must put the nearby HQS into motion. The SCC expels the magnetic field or confines it in the form of quantized fluxons by the Meissner effect, involving them by a vortex of a quantized screening velocity field (screening currents). In its turn, the HQS cannot expel the matter fields, but confines them involving them by a velocity field of the HQS and thereby giving inertial mass to the particles. While the velocity field of the SCC creates a reaction Lorentz force field thrusting the magnetic field, the velocity field of the HQS creates a reaction inertial force field thrusting the elementary particles.

Any excitation in the SCC becomes persistent because the SCC is perfectly conservative and opposes it to any new phase displacement. This is electromagnetic inertia, related with the inertial mass of photons confined within the SC. Analogously, any excitation in the HQS becomes persistent, which however is mechanical inertia related with the inertial mass of the elementary particles. This persistence or mechanical inertia underlies the conservation of the linear and angular momentum of isolated matter systems. However, while excitations in the SCC involve about $1 \mathrm{meV}$ of energy in the $\mathrm{HC}$ they involve typically hundreds of $\mathrm{GeV}$.

Any local excitation in a quantum fluid by an interacting field costs energy, because it must conquer with the phase correlation and locally increase the potential energy. The presence of an EM field in a superconductor induces macroscopic screening currents, elevating the energy of the SCC. The SCC can lower its energy by developing a macroscopic Lorentz reaction force field, compressing or expelling the magnetic field out from the superconductor. This is the macroscopic Meissner effect. The presence of a matter body in the HQS too may locally induce a macroscopic screening velocity field of the HQS, increasing the energy of the HC. The HQS too may develop a macroscopic reaction force field. However, this macroscopic reaction force field is an inertial force field thrusting and compressing the matter fields into regions of space where the Higgs order parameter already has been weakened by a large concentration of matter. This inertial force field is a macroscopic manifestation of the Higgs mechanism that will be shown in Section VI to correspond exactly to a gravitational force field.

From the viewpoint of the HQS, the imaginary part of the wave functions of particles is related with the motion, which a particle, stationary in the ordinary space, undergoes, due to motion of the HQS itself. This motion cannot be described with respect to the ordinary space coordinates and must be expressed in terms of imaginary variables. In Section VI, such imaginary inertial motions within a Keplerian velocity field of the HQS will be seen to generate the gravitational acceleration. Actually, the Higgs theory needs a definitive confirmation. There however can hardly be question about the existence of a Higgs type mechanism, developed by a quantum fluid spatial medium that rules the inertial motion of matter and the propagation of light. 


\section{Troubles with the Special Theory of Relativity: Wrong Physics Making Apparently Right Predictions.}

According to Einstein's Special Theory of Relativity (STR), empty space contains nothing that can represent a reference for motions. In the view of this theory, the null results of the Michelson light anisotropy experiments demonstrate that distances and times have no absolute values. They change as a function of the relative velocity $\left(v_{r}\right)$ in such a way that the velocity of light is intrinsically isotropic and constant with respect to all possible relatively moving inertial references. In Einstein's view, from all the possible methods of measuring the velocity of light within a given inertial reference (IR), the most precise is the one of light go-return round-trips along a known distance and clock method.

Clocks count time in terms of the oscillation period of a time standard, which may be a classical or a quantum oscillator. Actually, the best time standards, used in the atomic clocks, are the EM oscillations tuned to the frequency of the hyperfine transition of Cesium atoms. According to the STR, relative velocity with respect to the observer affects the frequency of such time standards in exactly the same proportion as it affects the light go-return round-trips. Lowering of the frequency of the radiation, emitted by hydrogen atoms, speeding at $n \times 10^{3} \mathrm{~km} / \mathrm{sec}(1 \leq n \leq 30)$ in the Ives-Stilwell experiments,[16] have been claimed to corroborate the predictions of the STR. Hence, measuring the velocity of light by the method of light go-return round-trips and clock, necessarily gives always the same result, regardless the relative velocity $\left(v_{r}\right)$ of the moving inertial reference, in this way apparently corroborating Einstein's postulate of the constancy of the velocity of light. In fact, the EM oscillations of the Cs atoms are go-return roundtrips of the EM field that are affected in the same proportion as light go-return roundtrips. Obviously the velocity of light, measured in this way, is an experimental artifact and not a property of light. Measuring the velocity of sound in the atmosphere by this same method, using an (open) sound-echo clock, too would give always the same result, regardless the strength of the wind. The velocity of sound, measured in this way, too is an experimental artifact.

With base in the postulate of the intrinsic constancy of the velocity of light, two clocks at different places in the same IR can be synchronized by the go-return roundtrips of light signals or very slow clock transport. In practice however, such an IR, valid in a large region of space, hardly exists in the universe. Therefore, from the viewpoint of the STR, true one-way measurements of the velocity of light are not possible, because synchronization of two relatively moving clocks at different places cannot be achieved.

From the viewpoint of the present work, the velocity of light has a well defined value $c_{H Q S}$ with respect to the HQS. Therefore, if Einstein's IR is moving with respect to the HQS, the velocity of light is anisotropic along this motion. In this case, two clocks, stationary in this inertial reference, can be synchronized by go-return light signals only along directions transverse to this motion. In practice, this is the case of an earth satellite; at the moment it passes vertically above the ground station, because, in this case, the up and down transit time of the signal is the same. In the case of polar orbits, good synchronization of the orbiting clocks to the ground clock can be achieved along the whole orbit, however only if the ground station is closely within the plane of the polar orbit.

Collective synchronization of the GPS clocks by Einstein's method, usually achieves $0.1 \mathrm{~ns}$ (time for light to travel 3 $\mathrm{cm})$. For low orbit satellites, clock synchronization can even be up to an order of magnitude better. With the help of these tightly synchronized atomic clocks, the one-way velocity of electromagnetic (EM) signals (light) between satellites has been precisely measured. Especially clear-cut measurement of the one-way velocity of EM signals (light) was achieved between the robotic twin satellites of the Gravity Recovery and Climate Experiment (GRACE). [9] These twin satellites move in the same sense at nearly $8 \mathrm{~km} / \mathrm{sec}$ along coplanar and practically identical circular polar orbits at about $500 \mathrm{~km}$ of altitude, separated from each other by closely $200 \mathrm{~km}$ and their positions being monitored by the GPS within $3 \mathrm{~cm}$. In order to measure microgravity effects, these satellites need to be equipped with highly stable atomic clocks, synchronized to better than $0.16 \mathrm{~ns}$.

It is observed that the signal transit time from the leading satellite to the rear satellite corresponds to a shortening by more than five meters $(17 n s)$ with respect to the expectations of the STR, while the signal transit time from the rear satellite to the leading satellite is lengthened by more than five meters $(17 n s)$. These discrepancies correspond in both cases exactly to the distance moved by the receiving satellite during the signal transit time and are consistent with backward signal anisotropy of nearly $8 \mathrm{~km} / \mathrm{sec}$ with respect to the satellites. This anisotropy is two orders of magnitude larger than the experimental precision of the experiment and shows that the EM signal (light) has a well-defined and isotropic NorthSouth velocity $(c)$ within the geostatic non-rotating reference, the same with respect to which the satellites are moving at 8 $\mathrm{km} / \mathrm{sec}$.

This one-way anisotropy of light unambiguously proves, by the first time, that a spatial medium exists (possibly the Higgs Quantum Space) that propagates light at the characteristic constant velocity $c$ with respect to this medium. This also shows that the medium propagating light is not moving along the North-South direction with respect to Earth. This anisotropy is due exclusively to the motion of the satellites and hence no additional effects need to be considered. This observation clearly and conclusively breaks by the first time the century old believe that the velocity of light is intrinsically constant and isotropic with respect to all possible inertial references.

The immediate consequence of this one-way anisotropy of the velocity of light is the absolute need of revising the interpretation of all the light anisotropy experiments performed in the past century. Most of the Michelson experiments measured the light anisotropy, due to the orbital and cosmic motion of earth. Systematically all these experiments obtained nominally null results. Now this must be interpreted as proving that the earth globe has no resultant velocity with respect to the HQS propagating light. Obviously this can make a sense only if the HQS moves with earth round the sun and with the solar system round the galactic center etc.

Some Michelson light anisotropy experiments, using highly sensitive Michelson interferometers rotating within the earth-based laboratories measured the anisotropy of light with respect to the laboratory itself. They all found small positive anisotropies of about $8 \mathrm{~km} / \mathrm{sec}$, nearly constant the whole day and the whole year. Fig. 2 displays the most com- 
plete data by D. Miller. [17]

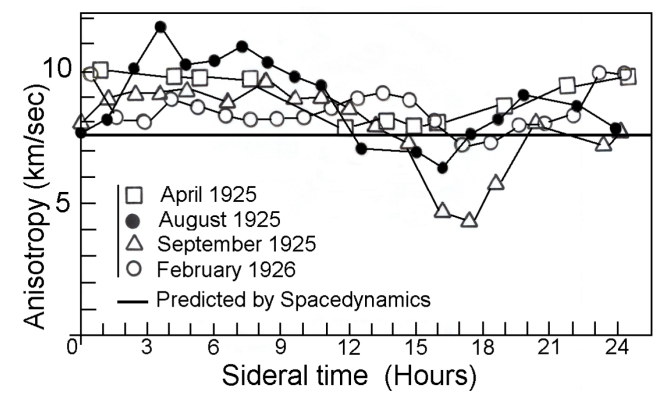

Figure 2. Constant nearly West-East anisotropy of light with respect to the earth based laboratories, by D. Miller.[17]

These anisotropy results give evidence that the HQS is moving through the earth based laboratories in the sense of the Moon's orbital motion at nearly $8 \mathrm{~km} / \mathrm{sec}$. As this anisotropy is much lower than expected from the ether theories, it was seen as flawed or simply ignored.

According to the above light anisotropy experiments and other recent even more persuasive experimental observations $[10,11]$, to be discussed in the next Section V, the HQS, ruling the inertial motion of matter and propagating light, is circulating round earth, round the sun and round the galactic center, according to velocity fields consistent with the respective local main astronomical motions. [12, 13, 14] Hence, earth is very closely stationary with respect to the moving HQS in the solar system and within the galaxy. This directly explains the null results of the Michelson light anisotropy experiments that searched for effects of the orbital and cosmic motion of earth. Hence, the reason of the null results of the Michelson light anisotropy experiments, is not the intrinsic constancy of light, but simply reveals the true kinematical circumstance of earth with respect to the HQS, propagating light. However, the velocity fields of the HQS round earth, round the sun and round the galactic center, consistently with the local main astronomical motions, besides explaining the null results of the light anisotropy experiments, they play a much more fundamental role. They appropriately create the gravitational dynamics on earth, in the solar system and within the galaxy. A summary of this gravitational mechanism will be given in Section VI (see also references $[12,13,14])$. Within this view, the null results of the Michelson light anisotropy experiments are exactly the signature of the physical mechanism of gravity in action that Einstein has missed.

The only relevant velocity of the earth-based laboratories with respect to the HQS is the one due to the local earth's Keplerian velocity field, generating the gravitational dynamics within the earth's field. This velocity field achieves nearly $8 \mathrm{~km} / \mathrm{sec}$ on surface and its effect on light and clocks is in the order of $10^{-10}$. Only Mössbauer experiments,[18], measuring the gravitational time dilation, and some very sensitive light anisotropy experiments[17] have been able to detect such low effects. Only very recently have the experimental techniques, with the help of atomic clocks, achieved sensitivity enough to measure consistently the low effects, due to the velocity of HQS through the earth based laboratories.

It is important to note that, within the experimental precision of the Ives-Stilwell experiment,[16] the effects of the large velocity of the hydrogen atoms $\left(n \times 10^{3} \mathrm{~km} / \mathrm{sec}, 1 \leq\right.$ $n \leq 30$ ), with respect to the earth-based laboratories, cannot be distinguished from the effects of their almost equally large velocity with respect to the HQS $\left(n \times 10^{3} \pm 8 \mathrm{~km} / \mathrm{sec}\right)$. Therefore, the results of the Ives-Stilwell experiment can equally well be seen as corroborating the time dilation, due to velocity with respect to the moving HQS. However, without ambiguity in this case (please see below). From the HQS point of view, the velocity of light has a well defined value $c_{H Q S}$ with respect to the HQS and not with respect to all inertial references. In fact, the light go-return round trips and the time standards by which clocks count time are affected in the same proportion by velocity of the laboratory with respect to the HQS. Hence, measuring the velocity of light by the light go-return round-trip and clock method too gives always the same value, independently from the velocity of the laboratory with respect to the HQS and too is an experimental artifact. Besides this, note also that the simple presence of the $\mathrm{HC}$, filling up the ordinary space is by it alone in clear conflict with the absolutely empty space of the TR. This HQS is not only a reference for the motions of particles and for the propagation of light, but literally rules these motions.

It is obviously important to note that all the conventional experimental tests of the STR were performed with atoms and or elementary particles moving at very high velocities with respect to the earth-based laboratories. Usually the results of these experiments are claimed to corroborate the STR. In fact, however the results of these experiments only apparently match the predictions of the STR. The reason is that the earth-based laboratories move only at nearly $8 \mathrm{~km} / \mathrm{sec}$ with respect to the HQS. The effect of such low velocity is in the order of $10^{-10}$ and hence totally imperceptible within the precision of these experiments. Coincidently, the earth-based laboratories also accomplish very closely the kinematic circumstances of the hypothetically resting observer in all of Einstein's thought experiments. These laboratories almost really rest with respect to the HQS. Therefore, the observed effects, due to the very large velocity $(\sim c)$ of the testing bodies (atoms or elementary particles) with respect to the earth-based laboratories, are in fact simply the effects of the equally large velocity with respect to the local HQS. These experimental results certainly are true. What is wrong, is interpreting them as if an earth-based laboratory were a generic reference. In fact, an earth based laboratory is a very specific preferred reference that nearly rests with respect to the local HQS. This blunder, committed in all the experimental tests of the STR, is analogous to postulating that the atmospheric pressure and the velocity of sound are universal constants throughout the universe and intending confirmation by measuring them in a laboratory, installed in the hold of a ship, navigating round the world.

To say that the fundamental assumptions of the STR are false and that nevertheless most of its predictions apparently match the experimental observations within earth-based laboratories could seem stupid. However, this historical relativistic blunder involves two errors, one error giving support to the other. Einstein's error has begun with a wrong interpretation of the null results of the Michelson light anisotropy experiments. This has led him to a wrong view about the nature of space. The wrong nature of space has led him to deny any intrinsic meaning of the motions and to impute all the effects of motions to relative velocity. The scientific community, on testing the predictions of the STR, committed the second error, considering the earth based laboratories as generic references. In fact, the earth-based laboratories are very specific preferred references that almost truly rest with 
respect to the moving HQS, ruling the motion of matter and propagating light. Interestingly, motion of the HQS according to a Keplerian velocity field, consistently with the local main astronomical motions, creates the gravitational fields. What is difficult to understand is why, after a whole century of impasses, some people still defend the relativistic view.

The above facts all-together inexorably force us to conclude that the true cause of all the so called relativistic effects is not relative velocity, however velocity with respect to the moving HQS, ruling the inertial motion of matter and propagating light. The fact that, within the conditions of the experimental tests of the STR, high velocities with respect to the earth based laboratories are almost equally high velocities with respect to the HQS, has hidden Einstein's wrong interpretation of the null results of the Michelson light anisotropy experiments and all the conclusions thereon. Actually, however, the experimental techniques, involving the tightly and collectively synchronized atomic clocks in orbit, are precise enough to disclose the relativistic blunder. In order to amend this error, it is sufficient to replace the relative velocity $v_{r}$, with respect to the observer, by the velocity $v_{H Q S}$, with respect to the HQS and the hypothetically constant velocity of light $c$ (experimental artifact) by the constant velocity of light $c_{H Q S}$ with respect to the HQS in all of Einstein's expressions for time dilation, relativistic mass and the relativistic energy (please see also reference [14] for more details). While this constitutes a radical dump of the fundamental assumptions of the STR about the nature of space and about motions, the changes in all of the practical outcomes, including Dirac's relativistic quantum theory of the electron, are totally irrelevant. This however in no way will say that the inferences of the HQS-dynamics are unimportant. Making precise predictions is obviously important. However, making these predictions with base in true physical causes is vital. For instance, Newton's theory of gravitation makes very precise predictions about circular orbital motions of the planets and notwithstanding these predictions are worthless, because of the simple reason that the gravitational forces cannot be real. They are fictitious. The STR clearly has been constructed on a wrong conceptual basis that entails wrong views about the nature of space and about the physics of motion. The impact of the inferences of the HQS-dynamics on the conceptual aspects of the gravitational physics (next section) too is enormous.

In conclusion, the effects of motion on clocks, on the mass and on the energy of particles, appointed by the STR, are real. The STR even gives their right dependence on velocity. However, these effects are ascribed to the wrong velocity with respect to the wrong references. The motions responsible for these effects are the velocities with respect to the HQS. In the scenario of the moving HQS, relative velocity between particles has only locally a simple meaning.

\section{Troubles with the General Theory of Relativity.}

While, on one hand, the Higgs mechanism explains the origin of the inertial mass and of the inertial behavior of the elementary particles, on the other hand, it provides us with the basis to disclose the genuine physical mechanism of gravity. According to Einstein's principle of equivalence, gravitational and inertial effects are equivalent and hence the grav- itational pull must be seen as an inertial pull. The next Section VI will show in some detail how this equivalence may be put straightforwardly in practice to generate the gravitational dynamics. Unfortunately, Michelson, Lorentz, Einstein and scientists along a whole century have not perceived that the null results of the Michelson light anisotropy experiments are exactly a genuine signature of the true physical mechanism of gravity in action. The wrong interpretation of the null results of the Michelson light anisotropy experiments has closed the possibility of putting the principle of equivalence directly in practice. Einstein's absolutely empty space (vacuum) is idle and can play no active role in the gravitational dynamics. Therefore he has conceived the motion of free bodies within the gravitational fields as generalized inertial motions along geodesic paths in a hypothetical curved fourdimensional spacetime that is impossible to picture. Note however that the curved spacetime is not the same thing as the Keplerian velocity field of the HQS.

The curved spacetime of GR can explain the free-fall on earth and predict the orbital motions of the planets as well as several effects of the gravitational fields on the propagation of light and on the rate of clocks. Now however the Theory of Relativity (TR) cannot explain an increasing number of clearcut experimental observations achieved with the help of the tightly synchronized clocks in orbit round earth.[9, 10, 11] These observations demonstrate that the TR and GR symmetrize space and the gravitational fields too much, thereby eliminating anisotropies that really exist and now, with the help of the new highly sensitive experimental techniques, achieved with the help of the tightly synchronized atomic clocks in orbit, are being discovered.

The most lethal experimental observation to GR is the absence of the gravitational slowing of the GPS clocks, that is predicted by GR, but not observed. According to GR, the gravitational time dilation, due to a gravitational potential $U$ is given by $T=T_{0}\left(1-2 U / c^{2}\right)^{-1 / 2}$, where $T_{0}$ is the time under $U=0$. To first order, the predicted slowing of the clocks is proportional to $U / c^{2}$. Hence, the effect of the solar gravitational potential on the GPS clocks, having orbital plane closely parallel to the earth-sun axis, during the 6 hours closer from the sun, should cause a total delay of more than $24 n s$, which would be recovered during the 6 hours farther from the sun. The corresponding 12 hours periodic sinusoidal variation in the time display of the GPS clocks would be more than two orders of magnitude larger than the stability and precision of these clocks within this period. However, observations show no sign of such variation. $[10,11]$ GR cannot explain this absence because the gravitational potential is a scalar.

Some people claim that the absence of the gravitational time dilation on the GPS clocks is due to cancellation by special relativistic time dilation. However, a simple calculation shows that special relativistic effects, due to the variation of velocity of the GPS satellites within the solar non-rotating reference, would be three orders of magnitude larger than those, due to the solar gravitational potential would and too are not observed. Others [19] claim that the absence is because the GPS satellites together with earth are free falling in the solar gravitational field. However, within this view, these same GPS satellites are also free falling in the earth's gravitational field and notwithstanding show clearly the slowing by the earthâĂŹs gravitational field of $(8 / c)^{2} \mathrm{sec} / \mathrm{sec}$, where $c$ is in $\mathrm{km} / \mathrm{sec}$. Please see Section VII.9 for the exact rea- 
son. Besides this, experiments with Muons in cyclotrons have shown that accelerations up to $10^{19} \mathrm{~m} / \mathrm{sec}^{2}$ cause no effect on the mean life time of the Muons and hence no time dilation.[20]

On one hand, the gravitational slowing of the GPS clocks by the solar gravitational potential, which is predicted by GR, but not observed $[10,11]$ has very drastic consequences on the current views about the nature of space and of the gravitational fields. On the other hand, it fully corroborates the new interpretation of the light anisotropy experiments proposed in the above Section IV.

Time dilation is well known to be caused by motion as evidenced by the well known increased lifetimes of speeding Muons and the red shifts of the radiation emitted by speeding Hydrogen atoms [16]. In the STR this time dilation effect is imputed to the relative velocity $\left(v_{r}\right)$ with respect to the hypothetically stationary laboratory observer. To first approximation this effect is proportional to $\left(v_{r} / c\right)^{2}$. Obviously, the observed gravitational slowing of the atomic clocks within the earth based laboratories cannot be due to relative velocity, because these clocks rest with respect to the laboratory observer. Likewise clocks stationary on earth, clocks stationary in the solar gravitational field, with respect to the solar nonrotating coordinates, certainly would be slowed by $(30 / c)^{2}$ ( $\mathrm{c}$ in $\mathrm{km} / \mathrm{sec}$ ). However, if motions cause time dilation, why then does the orbital motion of earth suppress the time dilation, due to the solar gravitational field, on the GPS clocks? Absurdly in one case, motion causes time dilation and in the other case, it suppresses it. This flagrant contradiction lets clear that the gravitational slowing of the clocks on earth is not caused by the gravitational potential. However, it also cannot be caused by relative velocity. The only reasonable conclusion is that the gravitational slowing of the atomic clocks, stationary in earth based laboratories, is caused by a velocity of a more fundamental nature that will be identified as implicit velocity with respect to the HQS. This singles out velocity with respect to the HQS as the unified cause of time dilation.

The observed slowing of the atomic clocks on earth, proportional to $(8 / \mathrm{c})^{2}$ (c in $\mathrm{km} / \mathrm{sec}$ ) must be related with the observed small constant anisotropy of light of nearly $8 \mathrm{~km} / \mathrm{sec}$ (please see Fig.2 and ref. [17]). Both these effects are observed within the earth based laboratories. Similarly, the absence of effects, due to the solar gravitational field, on the GPS clocks must be related with the absence of light anisotropy, due to the orbital motion of earth, as is well known. The mysterious implicit velocity $\left(V_{i m}\right)$ that causes the small light anisotropy of nearly $8 \mathrm{~km} / \mathrm{sec}$ within the earth based laboratories is the same that causes the observed small slowing of the clock rates on earth. On the other hand, the orbital motion of earth $(30 \mathrm{~km} / \mathrm{sec})$, which suppresses the gravitational time dilation, due to the solar gravitational field, also suppresses the light anisotropy due to the solar velocity field.

The only possible way to provide physical reality to this implicit velocity $V_{i m}$ is acknowledging the existence of a real spatial medium (HQS) that rules the inertial motion of matter and propagates light. This real spatial medium is moving in the ordinary three dimensions round earth (at $8 \mathrm{~km} / \mathrm{sec}$ on surface) in the sense of the Moon's orbital motion, as well as round the sun according to a velocity field consistent with the earth's orbital velocity $(30 \mathrm{~km} / \mathrm{sec})$ etc.

Visibly, within the velocity field of the HQS round the sun, earth is very closely stationary with respect to the circulating HQS. However, obviously earth cannot be kinematically privileged in detriment to all the other planets of the solar system and astronomical bodies in general throughout the universe. Earth is not the only planet commoving with the real HQS in the velocity field round the sun. All the planets must equally be closely commoving with the HQS. This will say that the HQS necessarily is moving according to a Keplerian velocity field round the sun:

$$
\vec{V}(r)=[\gamma M / r]^{1 / 2} \vec{e}_{\phi}
$$

where $\gamma$ is the Newtonian gravitational constant, $r$ is the radial spherical coordinate and $\vec{e}_{\phi}$ is the unit vector along the azimuthal spherical coordinate. This Keplerian velocity field is a cylindrical velocity field consistent with the local main astronomical motions, where the magnitude of the velocity for a given $r$ has the same value for all $\theta$ and all $\phi$ and hence its magnitude is spherically symmetric. This spherical symmetry assures the spherical symmetry of the gravitational fields.

The velocity field Eq.(3) will be shown to appropriately create the gravitational field of earth and of the sun and to naturally explain all the observations on the velocity of light and the rate of the clocks. It also fully corroborates the propositions made in Section IV concerning the null results odd the Michelson light anisotropy experiments. Such a Keplerian velocity field of the real HQS will be assumed to be circulating round each matter concentration throughout the universe, generating the respective gravitational fields.

The disk shape of the solar system and of the galaxies as well as of the satellites and planetary rings round planets all show that the orbits of the natural astronomical bodies in general are closely concentrated within the equatorial plane of the respective Keplerian velocity field. This minimizes their velocity with respect to the HQS. These orbital motions are almost totally determined by the circulation of the real HQS in the Keplerian velocity field and hence these bodies very closely rest with respect to the moving real HQS. This explains why the light anisotropy experiments, searching for light anisotropy due to the orbital and cosmic motions of earth, gave closely null results. The circular orbital motions of the planets need not to be explained anymore because it is space itself that so moves.

The very slow velocity of the planets of the solar system, in the order of only a few hundreds of $\mathrm{m} / \mathrm{sec}$, with respect to the moving real HQS is ruled by the principle of inertia. These very low velocities give rise to the very small deviations from the circular equatorial orbits that will be explained in Section VI. The vertical free fall is a case of extreme elliptic orbits in which the opposite implicit velocity $\left(V_{i m}\right)$ of the body with respect to real HQS just compensates the Keplerian velocity of the HQS.

The fact that this Keplerian velocity field of the HQS correctly creates the observed gravitational dynamics on earth, in the solar system and also generates correctly the galactic gravitational dynamics is very auspicious. However, it moreover correctly generates in terms of well known and genuine physics all the observed effects of the gravitational fields on the propagation of light and on the rate of clocks, including all the new effects recently discovered with the help of the GPS.

Einstein assumed that all the local inertial references (IRs) are free falling and that consequently the non-free falling 
earth based laboratories are accelerated upward. While on one hand this somehow introduces the idea of a local preferred reference that can move and accelerate, due to the spacetime curvature, on the other hand, it also leads to several difficulties. Why are these IRs free falling? What happens when they come to the gravitational center?

In conclusion, the absence of the gravitational time dilation, due to the solar gravitational field, which is predicted by GR but not observed, demonstrates that the HQS moves round earth, round the sun etc. according to Keplerian velocity fields, consistent with the local main astronomical motions. The present work replaces the curved space-time of GR by the Keplerian velocity field of the HQS (Eq.(3)) round each astronomical body throughout the universe. This velocity field is seen as a macroscopic manifestation of the Higgs mechanism confining and compressing the matter fields and thereby lowering its energy within the Higgs potential well (Fig.1). In this velocity field, the local inertial references are not free falling, but rotating round a fixed overhead axis. Hence, a particle, stationary with respect to ordinary space coordinates, is oppositely moving with respect to the HQS along a circular path in the local IR, round an overhead axis, under an upward centripetal force. This shows that the earth based laboratories effectively are accelerated upward as Einstein guessed. However, the nature of this upward acceleration is centripetal. The local upward centripetal acceleration involves a completely different dynamics of the space (HQS), ruling the inertial motion, which is free from all the troubles of Einstein's free-falling IRs.

The Keplerian velocity field will be shown to directly lead to the genuine physical mechanism of gravity as observed on earth and in the solar system. In references [12, 13], it is shown that this HQS-dynamics also can create the gravitational dynamics in the galaxies, without the need of dark matter. The planetary satellites are simply carried round the planets by the planetary Keplerian velocity field of the HQS along nearly circular equatorial orbits; the planets are carried round the sun by the solar Keplerian velocity field. These orbital motions need not to be explained anymore, because it is space itself that so moves. In the velocity field of the sun and of the galaxy, earth is very closely stationary with respect to the HQS, which explains the null results of the Michelson light anisotropy experiments. It also explains the observed absence of time dilation effects, due to the solar gravitational field, on the GPS clocks, which is predicted by GR but not observed. Section VII shows that this Keplerian velocity field of the HQS also predicts appropriately all the effects, caused by the gravitational fields on the propagation of light and on the rate of clocks. The conclusion is that the astronomical motions throughout the universe closely follow the motion of the HQS, so that celestial mechanics is very closely dual to the HQS-dynamics. Reference [21] provides additional evidence for this conclusion.

The theory of Quantum Gravity (QG) tries to reproduce the scenario of a central field of the hypothetical Newtonian gravitational forces in terms of the exchange of virtual field quanta (gravitons) emitted by the gravitational fields of astronomical bodies. The trouble of QG is the unlimited graviton-graviton interaction, leading to non-renormalizable divergences and preventing it from making testable predictions. Moreover, likewise GR, it too cannot explain the recent experimental observations, obtained with the help of the atomic clocks in orbit.

\section{Gravitational Dynamics as Result of Matter Wave Propagation Within the Keplerian Velocity Field of the Higgs Quantum Space.}

In this section, the rules of matter wave propagation (inertia), outlined in the introduction and completed in Section III, will be put in practice, within the Keplerian velocity field of the HQS round earth and round the sun, creating the respective gravitational fields. Details of this gravitational mechanism have been described in Reference [12] and [22]. Here only a summary will be given. In order to achieve a precise description, consider systems of non-rotating orthogonal (XYZ) coordinate axes, directions fixed with respect to distant objects in the universe, with origins fixed respectively to the centers of earth and of sun and the $\mathrm{Z}$ axes pointing along the rotation axes of earth and of the solar system, which also are the rotation axes of the HQS velocity field. Let $(r, \theta, \phi)$ be the usual spherical coordinates and $\left(\vec{e}_{r}, \vec{e}_{\theta}, \vec{e}_{\phi}\right)$ the unit vectors along the respective spherical coordinates.

VI.1. The gravitational dynamics on earth and within the solar system.

The Keplerian velocity field of the HQS, $\vec{V}(r)=$ $(\gamma M / r)^{1 / 2} \overrightarrow{\mathbf{e}}_{\phi}$ (see Eq.(3)) is a cylindrical velocity field along the $\phi$ spherical coordinate, where the magnitude of the velocity is spherically symmetric. A body stationary with respect to the local HQS at the equator of the velocity field simply follows the circular motion of the HQS. Despite the circular orbital motion, such a body is locally motionless with respect to the HQS and physically undergoes no acceleration. The circular equatorial orbital motion of such bodies need not to be explained anymore, because it is the HQS itself, the ultimate reference for rest and for motions, that is so moving and carrying them around. The circular equatorial orbital motion of the planets round the sun and the orbital motion of the Moon round the earth track very closely the motion of the HQS itself. This directly explains the null results of the Michelson light anisotropy experiments as well as the absence of the gravitational time dilation of the GPS clocks, due to the solar gravitational field. In fact the planets move only very slowly (about $100 \mathrm{~m} / \mathrm{sec}$ ) with respect to the local HQS, which causes the small ellipticity of their orbits. Effects of such low velocity on light and clocks is much too low to be detected even by the actual most precise measuring techniques.

A particle, fixed with respect to the (X,Y,Z) axes at any point $[r, \theta, \phi]$ (outside the source) within this Keplerian velocity field will be moving with respect to the HQS with the implicit velocity:

$$
\vec{V}_{i m p}(r)=-(\gamma M / r)^{1 / 2} \overrightarrow{\mathbf{e}}_{\phi}
$$

Note the negative sign in the right-hand side of this equation. The velocity of such a particle is implicit (Imaginary) because it cannot be specified with respect to the ordinary space coordinate $[\mathrm{X}, \mathrm{Y}, \mathrm{Z}]$ axes. The wave-fronts of this particle lie in the $[r, \theta]$ plain and, as the velocity of HQS along $+\phi$ increases for decreasing $r$, they see a velocity distribution that corresponds to a rotation of the local HQS and of the lo- 
cal Inertial Reference (IR) (a different one at each point of space) round an overhead axis. The corresponding angular velocity vector $\vec{W}$ is imaginary too (value and position to be found) and points along $+\theta$. This will say that the particle of mass $m$, resting with respect to the (XYZ) axes, is implicitly moving oppositely within the local rotating IR along a circular path round this same overhead axis and, in order to do so, a real upward centripetal force $(m g)$ must be acting on it. Otherwise, the particle will move along an instantaneous straight line within each local rotating IR, developing a real vertical downward velocity component, which the observer in the fixed non-inertial laboratory interbreeds as free-fall.

The HQS is of course not rotating round an overhead axis, but circulates round the gravitational center. The rotation of the local-IR about the overhead axis is real only to the wave fronts of the particle in the $[r, \theta]$ plane. However, the wave fronts of a particle, having a velocity component only along the $r$ coordinate, lying in the $[\theta, \phi]$ plane, see an opposite rotation of the HQS round the gravitational center. Totally similar refraction effects occur for sound waves propagating within wind gradients or within whirlwind.[23] Finally the wave fronts of a particle, having a velocity component only along the polar coordinate, that lie in the $[r, \phi]$ plane, are not refracted at all, because the Keplerian velocity field has no velocity component normal to the $[r, \phi]$ plane.

The opposite rotations (refraction) of the implicit (Imaginary) $\phi$ velocity component and of the ordinary (Real) $r$ velocity component of the particle characterizes a hyperbolic rotation. Note also that the HQS in the Keplerian velocity field contracts along certain directions and stretches along others, thereby changing the wavelengths of the particles along these directions and hence their velocity with respect to the HQS, according to de Broglie's equation $p=h / \lambda$. However, along the $r$ and the $\phi$ directions no such deformations occur and hence, if the effects of the Keplerian velocity field on the motion of the particle wave-packets are expressed in terms of the effective rotation rates of the $\phi$ and $r$ velocity components these effects are automatically taken into account and need not to be included explicitly.

In order to find the effective refraction rates of the $\phi$ and the $r$ velocity components of the particle in the Keplerian velocity field, the velocity of a small test body along an elliptic equatorial orbit (eccentricity 0.5 ) round a large gravitational source $M$ has been calculated to high precision. Knowing the orbital velocity $\vec{v}_{\text {orb }}$ and the velocity of the HQS $\vec{V}(r)$, given by equation (3) in the Keplerian velocity field, the propagation velocity $\vec{v}_{\text {prop }}$ of the particle can be found, using the relation $\vec{V}(r)+\vec{v}_{\text {prop }}=\vec{v}_{\text {orb }}$. The orbital, field and propagation velocities were plotted at a number of positions along the orbit. On these plots, the effective refraction rates of the $r$ and $\phi$ velocity components of $\vec{v}_{\text {prop }}$ can be read precisely enough at the orbital positions where the propagation velocity has only a $\phi$ or only a $r$ component. Please see full details in References [13] and [21]. The obtained rotation rates are:

$$
\begin{aligned}
\vec{W}_{r}(r) & =-\frac{1}{2}\left[\gamma M / r^{3}\right]^{1 / 2} \vec{e}_{\theta} \\
\vec{W}_{\phi}(r) & =\left[\gamma M / r^{3}\right]^{1 / 2} \vec{e}_{\theta} \\
\vec{W}_{\theta}(r) & =0
\end{aligned}
$$

Note that the rate of the $r$ velocity component, given by Eq. (5a), is only one half the rate Eq. (5b) of the $\phi$ component. This accomplishes the Virial theorem and assures conservation of the total mechanical energy. The refraction rate of the $r$ velocity component (Eq.(5a) ) just compensates for the variation of the velocity field as a function of $r$. This assures the conservation of the angular momentum round the center of mass of the rotating system and constraints the path of a free-falling particle from initial rest to be strictly along the radial coordinate, as observed.

In terms of rotation rate Eq.(5b) and the implicit velocity Eq.(4), the instantaneous rate at which vertical downward velocity is generated on a free-particle, on from initial rest with respect to the (XYZ) axes, in the field of a large body with mass $M$, is given by the vector product:

$$
\vec{g}(r)=\vec{W}_{\phi}(r) \times \vec{V}_{i m p}(r)=-\gamma M / r^{2} \overrightarrow{\mathbf{e}}_{r}
$$

This acceleration toward the gravitational center does not depend on the angular coordinates. It is a spherically symmetric field of acceleration toward the gravitational center.

In the case of two interacting masses $M_{1}$ and $M_{2}$, equation (6) must be written for both sources $M_{1}$ as well as for $M_{2}$ and inserting the other mass. In this case $r$ is the distance between the two masses. If the two masses are orbiting round the center of mass (binary), $\vec{V}_{i m p}=0$. In this case, there is physically no acceleration (no force) and the bodies simply move in the ordinary space together with the HQS, one in the other's velocity field round the center of mass. Note that in this case the orbital velocity is not equal to $v_{\text {orb }}=(\gamma M / r)^{1 / 2}$ but is a factor $\sqrt{1 / 2}$ lower. This feature is key to explain the non-Keplerian rotation rate of the galactic disk without the need of dark matter.[12] If the bodies are not orbiting at all, their implicit velocities are given by Eq.(4). Inserting $M_{2}$ into Eq.(6) for $M_{1}$ and $M_{1}$ into Eq.(6) for $M_{2}$, these expressions describe fictitious attractive forces toward the center of mass, which are equal and opposite. In fact the acceleration toward the center of mass is due to the mutual influence on the respective local inertial references.

Equation (6) is precise only for free-fall along short distances. The free-fall along large distances is described by the elementary homogeneous linear differential equation:

$$
\frac{d \vec{\nu}}{d t}=\mathbf{A} \vec{\nu}
$$

where $\vec{\nu}$ is the column matrix of the $r$ and the $\phi$ velocity components:

$$
\vec{\nu}(t)=\left(\begin{array}{c}
\nu_{r}(t) \\
\nu_{\phi}(t)
\end{array}\right)
$$

and $\mathbf{A}$ is the hyperbolic infinitesimal rotation matrix, defined in terms of the rotation rates, given in Eqs.(5):

$$
\mathbf{A}=\left(\begin{array}{cc}
0 & W_{\phi} d t \\
-W_{r} d t & 0
\end{array}\right)=\left(\begin{array}{cc}
0 & W d t \\
\frac{1}{u} W d t & 0
\end{array}\right)
$$

where $u=2 M /(M+m)$ accounts for the asymmetric distribution of kinetic energy between $m$ and $M$ as well as for the explicit time dependence of the velocity field $\vec{V}(r)$ due to motions of the source $M$ with respect to the center of mass under the field of $m$. Inserting $\nu$ and $A$, defined by equations (8) and (9), into equation (7) develops into Equation (10): 


$$
\begin{aligned}
\vec{\nu}(t) & =\vec{\nu}_{0} \operatorname{Exp}\left[\int_{0}^{t} A\left(r\left(t^{\prime}\right)\right) d t^{\prime}\right] \\
& =\sum_{n=0}^{\infty} \frac{1}{n !}\left(\begin{array}{cc}
0 & \Theta(t) \\
\frac{\Theta(t)}{u} & 0
\end{array}\right)^{n}\left(\begin{array}{c}
\nu_{r}(0) \\
\nu_{\phi}(0)
\end{array}\right) \\
& =\left(\begin{array}{cc}
\cosh \left(\frac{\Theta(t)}{\sqrt{u}}\right) & \sqrt{u} \sinh \left(\frac{\Theta(t)}{\sqrt{u}}\right) \\
\frac{1}{\sqrt{u}} \sinh \left(\frac{\Theta(t)}{\sqrt{u}}\right) & \cosh \left(\frac{\Theta(t)}{\sqrt{u}}\right)
\end{array}\right) \times\left(\begin{array}{l}
\nu_{r}(0) \\
\nu_{\phi}(0)
\end{array}\right)
\end{aligned}
$$

Note that this equation already characterizes clearly the (asymmetric) hyperbolic rotation. Equation (10) is a linear transformation of the Real $r$ and the Imaginary $\phi$ velocity components on going from one local rotating inertial reference to the other. This hyperbolic rotation is completely analogous to that of 4-vectors in the theory of relativity on going from one inertial reference to the other. Here nevertheless the parameter ruling the transformation is not relative velocity, however the characteristic local angular velocity $[\mathrm{W}(\mathrm{r})]$ of the local IRs.

For farther details of the solution of Eq.(7) please see $\operatorname{Refs}[14,21]$. The final solution, for free fall on from $r_{o}$ and initial rest $\nu_{r}(0)=0$, which means $\vec{\nu}_{\phi}(0)=\vec{V}_{i m p}\left(r_{0}\right)=$ $-\left(\gamma M / r_{0}\right)^{1 / 2} \overrightarrow{\mathbf{e}}_{\phi}$, the solution of Eq.(7) is:

$$
\begin{aligned}
& \nu_{r}(t)=\left[\frac{2 M}{M+m}\left(\frac{\gamma M}{r(t)}-\frac{\gamma M}{r_{0}}\right)\right]^{1 / 2} \\
& \nu_{\phi}(t)=V_{i m p}\left(r_{0}\right)\left[\frac{r_{0}}{r}\right]^{1 / 2}=\left[\frac{\gamma M}{r(t)}\right]^{1 / 2}
\end{aligned}
$$

Eq.(11a) is just the well known expression for the observed vertical free-fall on from rest at $r_{o}$ and Eq.(11b) is just the implicit (imaginary) velocity as a function of the radial position $r$, which shows that the refraction rate of the radial velocity component just compensates for the increase of the velocity field as a function of the decrease of the radial coordinate. This assures that the particle falls on from rest along a perfectly vertical (radial) path and hence assures conservation of the angular momentum about the gravitational center.

From the viewpoint of the ordinary space coordinates, the implicit velocity of a matter body $m$, falling in the field of $M$ ( $m<<M$ ), given by equation (11b), is an imaginary quantity $i \nu_{\phi}==>i\left[\frac{\gamma M}{r}\right]^{1 / 2}$. Its corresponding implicit kinetic energy $K(r)$ is:

$$
\begin{aligned}
K_{m}(r) & =\frac{1}{2} m \times(i)^{2} \nu_{\phi}^{2}=-(\cosh )^{2}\left[\frac{\Theta[r(t)]}{\sqrt{u}}\right]\left(V_{i m p}\right)^{2} \\
& =-\frac{1}{2} m \frac{\gamma M}{r}
\end{aligned}
$$

This negative quantity is an Imaginary kinetic energy term, which usually is interpreted as the potential energy associated with the body $m$ in the field of $M$. Potential energy in physics is an emergency concept to explain the ability of certain systems and or fields to absorb, store, conserve work and eventually giving it back. In reality, potential energy always involves at least two bodies. In the case of two gravitationally interacting bodies $m$ and $M, M$ too is free-falling in the field of $m$ and the corresponding (implicit kinetic) potential energy is:

$$
K_{M}(r)=-\frac{1}{2} M \frac{\gamma m}{r}
$$

Adding equations (12) and (13), which are identical in value, gives the total potential (implicit kinetic) energy $U(r)$ :

$$
U(r)=-\frac{\gamma m M}{r}
$$

The corresponding total Real kinetic energy $K$ is the sum of the kinetic energies of $m$ and $M$ with respect to the center of mass. However, if $(M » m)$, practically the whole kinetic energy is stored by $m$ and $u \approx 2$. This kinetic energy is given by:

$$
\left.K \approx \frac{1}{2} m \times 2(\gamma M) / r_{0}\right) \times \sinh ^{2}(\Theta / \sqrt{2})
$$

For details, please see Ref.[14].

Conservation of the total mechanical energy $E$ of a system of a free-falling body $m$ in the gravitational field of $M$ ( $m \ll M, u \approx 2$ ) may be expressed in terms of the hyperbolic sine and cosine as:

$$
\begin{aligned}
E[r(t)] & \approx-E[r(t=0)]\left[\cosh ^{2}\left[\frac{\Theta(t)}{\sqrt{2}}\right]-\sinh ^{2}\left[\frac{\Theta(t)}{\sqrt{2}}\right]\right] \\
& =\text { Constant }
\end{aligned}
$$

where the $\cosh ^{2}$ term is related with the potential energy and the $\sinh ^{2}$ term is related with the kinetic energy as a function of time.

\section{VI.2. Symmetry of the gravitational mechanism of spacedynamics with orbital motions.}

The orbital motion of the planets in principle cannot be looked anymore as motion, under a central field of gravitational forces in an inertial reference, valid in the whole region, because, within the Keplerian velocity field of the HQS (gravitational field), each local IR is valid rigorously only within an infinitesimal region of space. However, analyzing the motion of bodies along different orbits within the Keplerian velocity field of the HQS Eq.(3), it will become clear that the gravitational mechanism of spacedynamics is symmetric for direct and or retrograde orbital motions as well as for equatorial or polar orbits. This will be seen to simulate a central field of gravitational forces in an extended IR, exactly as conceived in Newtonian gravity.

Consider free-fall experiments within a small laboratory on the surface of a spherical rotating planet of radius $R$, whose angular velocity vector $\vec{w}$ coincides with the $Z$ axis of the velocity field of the HQS. A body, initially resting near to the surface of the rotating planet, will be moving with respect to the HQS at a propagation velocity:

$$
\begin{aligned}
\vec{\nu}(\theta) & =\vec{V}_{i m p}(R)+\vec{\nu}_{\text {rot }}(\theta) \\
& =-\left[(\gamma M / R)^{1 / 2} \mp \omega R \sin \theta\right] \overrightarrow{\mathbf{e}}_{\phi}
\end{aligned}
$$

where $\vec{V}_{i m p}$ is the implicit propagation velocity on the planet's surface for null angular velocity, given by Eq.(4), $\vec{\nu}_{\text {rot }}(\theta)$ is the local velocity with respect to the (XYZ) axes along the ordinary $\phi$ coordinate, due to the planet's rotation. This velocity depends on the latitude via $\sin \theta$ and the upper and lower signs are respectively for direct and retrograde rotation of the planet with respect to that of the velocity field of the HQS. 
If the body is free to move within the laboratory, then, besides the refraction rate given by Eq.(5b), the rotation of the planet necessarily changes the direction of this initial effective propagation velocity vector with respect to the gravitational center by an opposite trigonometric rotation rate, equal to the angular velocity of the planet. Hence, the effective rotation rate of the propagation velocity vector $\vec{\nu}(\theta)$ is:

$$
\begin{aligned}
\vec{\omega}_{\text {eff }}(\theta) & =\frac{1}{R}\left[V_{i m p} \pm\left(\nu_{\text {rot }}\right)\right] \overrightarrow{\mathbf{e}}_{\theta} \\
& =\left[\left(\gamma M / R^{3}\right)^{1 / 2} \pm \omega \sin \theta\right] \overrightarrow{\mathbf{e}}_{\theta}
\end{aligned}
$$

where the same convention for the upper and the lower signs as in Eq.(17) is used.

Considering the effective velocity (Eq.(17) and the effective angular velocity (Eq.(18), the effective gravitational acceleration on the planet's surface is:

$$
\vec{g}_{\text {eff }}(\theta)=\vec{\omega}_{\text {eff }} \times \vec{\nu}=-\left[\gamma M / R^{2}-\omega^{2} R \sin ^{2} \theta\right] \overrightarrow{\mathbf{e}}_{r}
$$

Eq. (19) shows that $\vec{g}_{\text {eff }}(\theta)$ is perfectly symmetric for direct or retrograde rotation of the planet (orbital motion of a body) and correctly gives the gravitational acceleration from the equator to the poles. The first term in the right hand side describes acceleration toward the gravitational center in the static situation [see Eq.(6)], while the second term describes an outward centrifugal acceleration.

For strictly circular polar orbits with radius $r>R, \vec{\nu}$ has velocity components along $\phi$ as well as along $\theta$ :

$$
\begin{gathered}
\vec{\nu}_{\phi}=\vec{V}_{i m p}(r)=-[\gamma M / r]^{1 / 2} \overrightarrow{\mathbf{e}}_{\phi} \\
\vec{\nu}_{\theta}= \pm[\gamma M / r]^{1 / 2} \overrightarrow{\mathbf{e}}_{\theta}
\end{gathered}
$$

While $\vec{\nu}_{\phi}$ generates the gravitational acceleration $\vec{g}(r)=$ $-\gamma M / r^{2} \overrightarrow{\mathbf{e}}_{r}$, see Eq.(6), the $\theta$ component is is not affected directly by spacedynamics, because the velocity field Eq.(3) has no velocity component along $\theta$.

$$
\vec{g}_{\text {eff }}(r)=-\left[\gamma M / r^{2}-\left(\nu_{\theta}^{2}\right) / r\right] \overrightarrow{\mathbf{e}}_{r}
$$

where the second term in the right hand side is a usual upward centrifugal effect due to the curved motion imposed by the first downward term. Depending on the value of the $\vec{\nu}_{\theta}$, the effect of the $\vec{\nu}_{\phi}$ component enforces the particle into a parabolic (downward elliptic), circular or (upward) elliptic path.

An analytical solution of Eq.(7) for more general motions is not at all easy. However, the results expressed by Eqs.(19) and (22) reveal a feature that circumvents this difficulty. These equations show that the effect of the HQS dynamics is completely independent from the effects of the ordinary dynamics. Clearly, the implicit dynamics is orthogonal to the dynamics in the ordinary space. While the HQS dynamics generates (simulates) the usual central field of Newtonian gravitational accelerations, the ordinary dynamics gives rise to the apparent centrifugal effects, due to the ordinary orbital motions, exactly as conceived in Newtonian gravitation. Hence, general motions in the spherically symmetric velocity field Eq.(3) can be treated as motions in an extended inertial reference, under a central field of fictitious gravitational forces. This explains why the Newtonian gravitation works so well.

\section{Effects of the Higgs Quantum Space Dynamics on the Propagation of Light and on the Rate of Clocks.}

The purpose of this Section is showing that the dynamics of the HQS, ruling the inertial motion of matter and the propagation of light, according to a Keplerian velocity field, consistent with the local main astronomical motions, thereby generating the gravitational fields, correctly predicts all the, so called and effectively observed relativistic effects. It however, predicts in addition all the effects on the propagation of light and the rate of the clocks, recently discovered with the help of the tightly synchronized atomic clocks in orbit, which the theory of relativity fails to explain.

VII-1. One-way Anisotropy of EM Signals Between Artificial Satellites.

The most precise measurements of the one-way anisotropy were obtained with the twin satellites of the GRACE project. These satellites were moving at $8 \mathrm{~km} / \mathrm{sec}$ along the same polar orbit at $500 \mathrm{~km}$ of altitude and separated by $200 \mathrm{~km}$. Simultaneous synchronization of their atomic clocks to the same reference clock on ground and close to the orbial plane of the satellites by Einstein's method, is very precise because the transit time of the up and down signals is very closely the same.[14] In the case of the twin satellites of the GRACE project, synchronization was better than $0.1 \mathrm{~ns}$. On exchanging signals between the twin satellites, it was observed that, while the signal transit time from the leading satellite to the rear satellite was shortened by more than $5 m(17 n s)$, the signal transit time from the rear satellite to the leading satellite was lengthened by $5 m$. [9] These values are consistent with a backward signal anisotropy of nearly $8 \mathrm{~km} / \mathrm{sec}$ with respect to the satellites, which is exactly the velocity of the satellites. This observation proves that a spatial medium (HQS), propagating the EM signals (light) at a well defined velocity $c_{H Q S}$ exists and that this medium is not moving along the NorthSouth direction, exactly as dictated by Eq.(3). The immediate consequence of this observation is the absolute need of revising the interpretation of all the light anisotropy experiments performed in the past century (see next Section).

\section{VII-2. Michelson light anisotropy experiments.}

The large majority of the Michelson experiments searched for light anisotropy, due to the orbital and cosmic motion of earth. They all found closely null results, which demonstrates that earth is very closely stationary with respect to the HQS, ruling the propagation of light. The absence of the gravitational time dilation on the GPS clocks, due to the solar gravitational potential, which is predicted by GR but not observed, perfectly corroborates this conclusion. The null anisotropy of light also shows that the velocity of our solar system in the Milky Way galaxy is consistent with the velocity field of the HQS that generates the galactic gravitational dynamics.[12] Moreover, the null anisotropy of light shows that the observed relative velocity of our galaxy, with respect to neighboring galaxies, as well as the velocity due to the recession between the galaxies too let earth stationary with respect to the HQS. This is possible only if the expansion of the universe is expansion of the HQS itself.[21] In the view of the present work, the only motion, that causes relevant anisotropy of light within the earth-based laboratories, is the local velocity field of the HQS round earth itself in the sense of the Moon's orbital motion and creating the 
earth's gravitational field. This velocity reaches $7.91 \mathrm{~km} / \mathrm{sec}$ on surface and is constant the whole day and the whole year. The most precise Michelson anisotropy experiments, searching for light anisotropy with respect to the earth based laboratories, found anisotropies closely below $8 \mathrm{~km} / \mathrm{sec}$, constant the whole day and the whole year [17] exactly as predicted by the HQS-dynamics (please see Fig.2)

VII-3. Gravitational time dilation and gravitational spectral red shifts.

The gravitational time dilation, slowing the clocks within the earth's field and the gravitational spectral red shifts are now well confirmed experimentally.[18, 24] To first approximation the effects predicted by the HQS-dynamics creating the gravitational fields, are proportional to $(\gamma M / r) / c_{H Q S}^{2}$, which also is predicted by GR, gives the observed values. In this expression $(\gamma M / r)^{1 / 2}$ is the velocity of the HQS through the experimental set ups. The nuclear, atomic and molecular energy states adjust them to these time dilation effects.

VII-4. Excess time delay of radar signals in round-trips within the solar system.

According to GR, heavy astronomical bodies cause changes of the spacetime geometry in their neighborhood. This lengthens the path of electromagnetic (EM) radiation passing closely by these bodies, giving rise to an excess time delay. Shapiro [25] has measured this excess time delay by time keeping the transit time of radar signals for complete go-return round-trips from earth to Venus and back to earth, during about two years. The excess time delays showed a cusp-like peak near the superior conjunction of Venus (see Fig.5), in conformity with the predictions of GR.

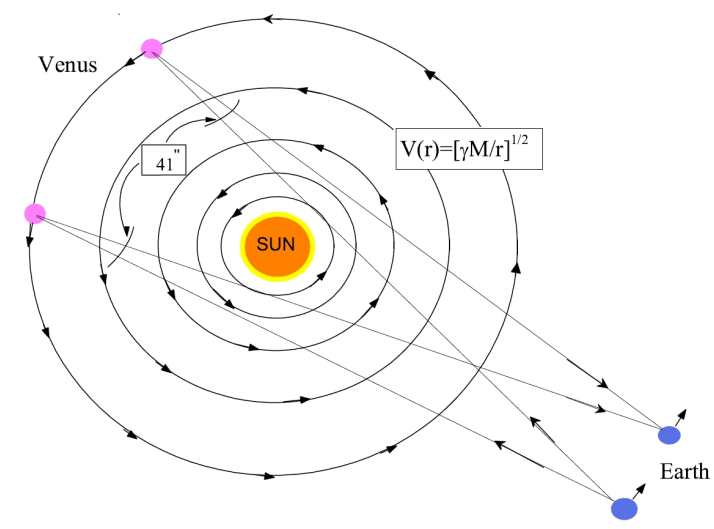

Figure 3. The path of radar signals from earth to Venus and back to earth within the Keplerian velocity field of the sun, before and after superior conjunction.

According to the present work the excess time delay is not caused by increase of the geometrical distance, however is due to the effective velocity $\vec{c}_{H Q S}+\vec{V}$ of the radar signals in the Keplerian velocity field of the HQS round the sun. Note that this HQS rules the velocity of propagation of the EM radiation. Hence, according to well known physics, while during the direct journey of the signal the velocity field is favorable (gain of time), during the retrograde journey it is unfavorable (loss of time). However, in this case it is well known that the loss of time is a little bit larger than the gain, so that an effective excess time delay results. The calculation of the excess time delay within the solar Keplerian velocity field is a little bit complicated because the effect varies as function of position and must be calculated by integration.

In the present work the excess time delay of the radar sig- nal round-trips was calculated separately for the go and for the return travel along straight paths from earth to Venus and back to earth, using the effective velocity $\vec{c}_{H Q S}+\vec{V}$. This calculation was repeated for a series of paths having minimum distances from the center of the sun (impact parameters) $\mathrm{R}=0,2,4,8,25,50$ and 100 million $\mathrm{km}$, before and after superior conjunction (please see Fig.3). The excess time delays were numerically calculated, dividing the straight line paths in a large number of segments (shorter segments close to the sun), computing the time-delay and time-gain, separately for the transverse and for the longitudinal components of the solar Keplerian velocity field along the signal paths and finally adding up (integrating) the contributions along the whole path. More than $99 \%$ of the excess time-delay or timegain comes from the longitudinal component of the velocity field along the path. In the calculation, the different EarthVenus distances, due to their orbital positions as well as the different signal path due to the motion of earth during the signal round-trips, was taken into account.

The results show that although the travel times for individual Earth-Venus and Venus-Earth journeys is considerably asymmetric, the effective excess time delay for the full roundtrips, displayed in Fig.4, practically coincide with the excess time delays measured by Shapiro,[25] and displayed in Fig.5. In the HQS-dynamics, this effective excess time delay is a simple and genuine physical effect, due to the effective signal velocity within the solar Keplerian velocity field of the HQS and not to the increase of the geometrical distance due to the arcane spacetime curvature, as asserted in GR. The fact that the Keplerian velocity field of the HQS round the sun correctly produces the effective excess time delay (Shapiro effect) provides strong support to the present HQS-dynamics gravitational mechanism.

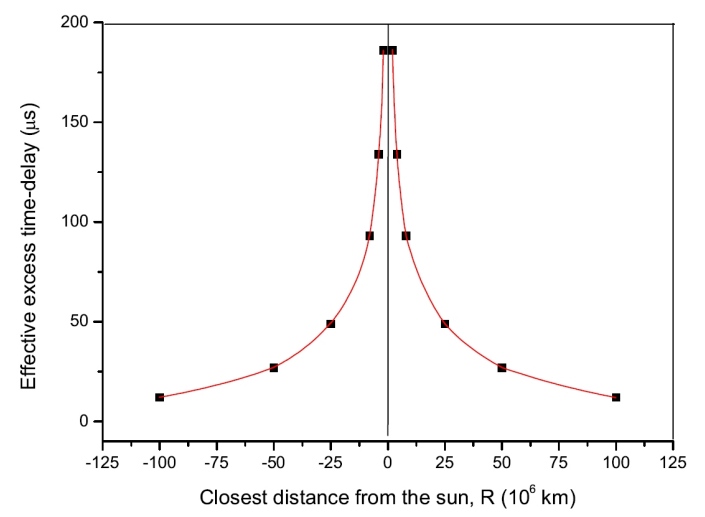

Figure 4. The effective excess time delay in microseconds ( $\mu s)$ (fourth column in Table I) for complete go-return travels, as calculated in terms of $\vec{c}_{H Q S}+\vec{V}$ within the solar Keplerian velocity field of the HQS.

Other even more precise measurements of the excess time delay (Shapiro Effect) of signals passing closely by the sun have been made with the help of Mars landed transponders during the Mariner 6 and Mariner 7 missions as well as other spacecrafts (Cassini). However, all these experiments measured the effective excess time delay for complete go-return signal roundtrips and hence could not detect the anisotropy in the velocity of the signals, displayed in Table 1.

\section{VII-5. The gravitational light lensing effect.}

Consider two light beams, propagating toward the sun along initially parallel paths by equidistant and opposite sides of the sun (please see Fig.6). First the wave vectors will be 
Table 1. The first column gives the values of the nominal impact parameters $R$, the second and third columns give the gain (-) and the excess delay (+) of time for respectively individual go and return journeys in milliseconds $(\mathrm{ms})$. Note the inversion of signs after superior conjunction of Venus. The forth and last column gives the effective excess time-delays for full go-return roundtrips in microseconds ( $\mu s$ ). Note the downward spike in the effective excess time delay for $R=0$. Shapiro could have seen this spike if the radar signals had passed closely above or below the sun.

\begin{tabular}{c|c|c|c}
$R\left(10^{6} \mathrm{~km}\right.$ units $)$ & $g o: \Delta t(\mathrm{~ms})$ & return $: \Delta t(\mathrm{~ms})$ & $\Delta t_{\text {eff }}(\mu \mathrm{s})$ \\
\hline 100 & -51.987 & 51.999 & 12 \\
\hline 50 & -76.697 & 76.725 & 28 \\
\hline 25 & -70.007 & 70.056 & 49 \\
\hline 8 & -48.585 & 48.679 & 94 \\
\hline 4 & -36.699 & 36.833 & 134 \\
\hline 2 & -27.165 & 27.351 & 186 \\
\hline 0 & 0.035 & 0.035 & 70 \\
\hline-2 & 27.351 & -27.165 & 186 \\
\hline-4 & 36.833 & -36.699 & 134 \\
\hline-8 & 48.679 & -48.585 & 94 \\
\hline-25 & 70.056 & -70.007 & 49 \\
\hline-50 & 76.725 & -76.697 & 28 \\
\hline-100 & 51.999 & -51.987 & 12
\end{tabular}

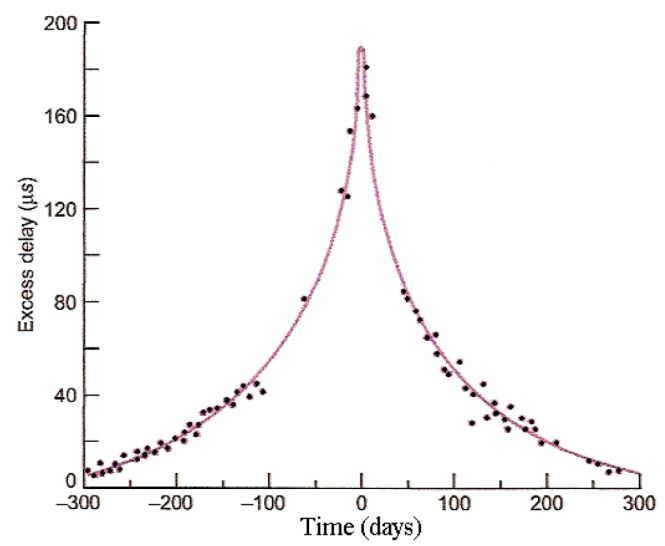

Figure 5. The effective excess time delay for a complete go-return roundtrip travels between earth and Venus as measured by Shapiro.[25] The value of $R= \pm 100 \times 10^{6} \mathrm{~km}$ in Fig.4 corresponds to about \pm 180 days in Fig. 5

refracted according to Eq.(5a) by a total angle $+\alpha$. The light beam gains a small velocity component $V(r)=(\gamma M / r)^{1 / 2}$ along $-\phi$ and reduces its radial velocity component to $c^{\prime}=$ $\left(c_{H Q S}^{2}-V^{2}\right)^{1 / 2}$. Near to the sun, where the solar velocity field achieves $436 \mathrm{~km} / \mathrm{sec}$, refraction by Eq.(5b) is dominant and the wave vector is refracted oppositely by a total angle $-2 \alpha$. Finally, after having passed the sun and going away at the opposite side, the wave vectors are refracted back according to Eq.(5a) by nearly an angle $+\alpha$. However, near to the sun, the solar velocity field is favorable to the prograde ray (left) so that it spends less time near to the sun and hence is deflected by a smaller angle $-(2 \alpha-\delta)$. To the retrograde ray (right) the solar velocity field is unfavorable and hence it spends a longer time near the sun and refracts by a larger angle $-(2 \alpha+\delta)$. This differentiated refraction $\delta$ by Eq.(5b) causes convergence of the rays that is responsible for the gravitational light lensing effect.

The excessive or insufficient deflection of light $\delta$ near the sun by the solar gravitational field can be calculated simply by multiplying the refraction rate Eq.(5b) times the excess (or shortage) of time delays listed in Table I. On the solar surface $R=6.9565 \cdot 10^{8} \mathrm{~m}$ and the refraction rate is $W_{\phi} \sim 0.0359 \mathrm{deg} / \mathrm{sec}$. Interpolating the value of $\Delta t$ in Table I for light passing by the surface of the sun, the total excess time delay at the retrograde side

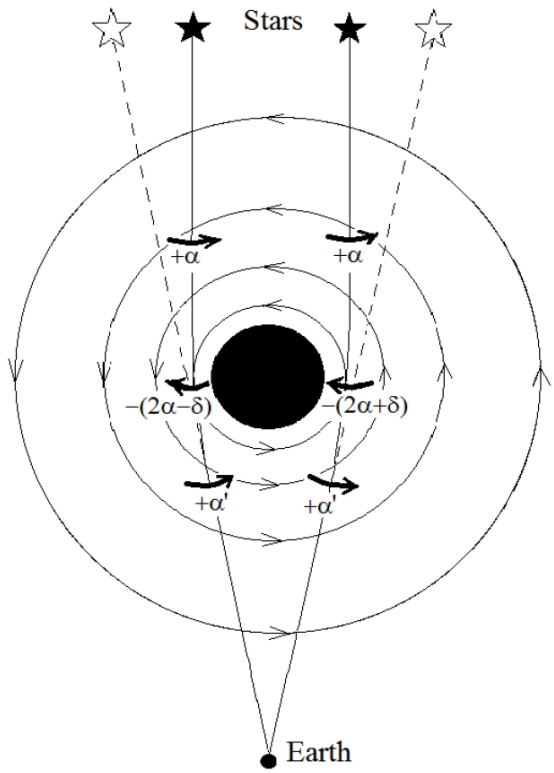

Figure 6. Gravitational light-lensing effect, by a heavy mass. The lightlensing results from the excessive deflection $\delta$ at the retrograde side (right) and the insufficient deflection $\delta$ at the prograde side (left).

gives closely $\Delta t=+13.5 \mathrm{~ms}$ or shortage at the prograde side $\Delta t=-13.5 \mathrm{~ms}$. The product $W_{\phi} \times \Delta t$ gives $\delta=1.745$ arcsec, which is very closely the observed value.

VII-6.The non-synchronous arrival of the pulsar signals to equidistant earthbased antennas along the Earth's orbital motion.

Astronomical observations with the help of interferometric methods can improve the images by orders of magnitude. This is achieved with the help of computers by coherently superposing the images (signals) received by separated telescopes or antennas, taking into account the distances from the observation field. The fundamental requisite for this method is that the signals received in the different observatories be synchronous. This is achieved by atomic clocks, closely synchronized with the help of the GPS clocks. On testing the synchrony of the clocks along the Very Long Base Line Interferometry set-ups with the arrival of the millisecond pulsar signals, a surprise. While the arrival to the equidistant 
terrestrial antennas along directions transverse to the earth's orbital motion was synchronous, along the orbital motion of earth the arrival was out of synchrony, up to 4.2 microseconds. This has raised a controversial about the synchronization of the clocks.[10, 11]

From the viewpoint of the HQS-dynamics, the wave fronts of signals coming from a distant pulsar toward the sun are refracted according to Eq.(5a). The angle of refraction is such that $\sin \beta=V / c_{H Q S}$, which is equal to the usual aberration angle of star light. Because of this refraction, the signal develops a velocity component $V(r)=(\gamma M / r)^{1 / 2}$ along $-\phi$ and reduces the radial velocity to $c^{\prime}=\left(c_{H Q S}^{2}-V^{2}\right)^{1 / 2}$.

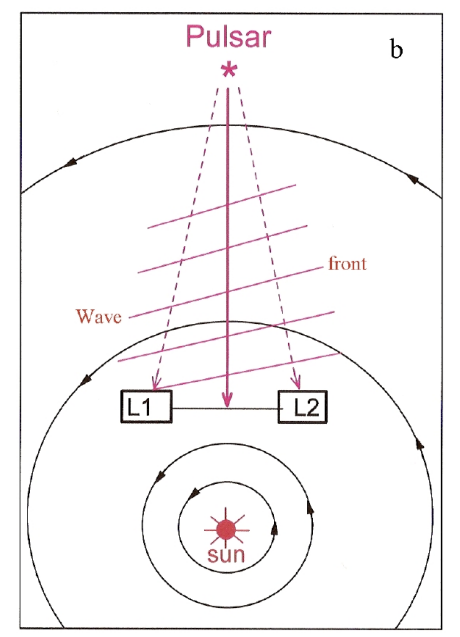

Figure 7. The wave fronts of an $E M$ signal coming from a distant pulsar toward the sun are slanted by an angle $\beta$, due to refraction according to Eq.(5a). Because of this slanting of the wave fronts, if the signal is detected by earth based antennas equidistant from the pulsar, and separeted by the earth's diameter, the wave front reaches first the leading antenna $L_{1}$ and only $4.2 \mu s$ later $L_{2}$. All effects are exaggerated in the figure to make them visible.

According to the HQS-dynamics, the non-synchronous arrival of the signal to the equidistant earth based observatories along the orbital motion of earth (please see Fig.7) is real and hence there is no reason for suspicions about the synchronization of the clocks in the distant observatories with the help of the GPS clocks. The fact that the observed arrival of the pulsar signal is exactly as dictated by Eqs.(5a) is a spectacular confirmation of the predictions of the HQS-dynamics.

\section{VII-7. The perihelion precession.}

An analogous differentiated refraction of the propagation velocity $\vec{v}$ of particles with respect to the HQS of an orbiting body as in the preceding Section VII-5 must be responsible for the perihelion precession of elliptical orbits. At the aphelion the direction of $\vec{v}$ is opposite to the velocity field (retrograde) as well as to the orbital motion. This increases the time of permanence in this region of the orbit. Hence, $\vec{v}$ refracts during a longer time. However, at the perihelion $\vec{v}$ is parallel to the velocity field (prograde), which displaces it more rapidly in the orbit, so that it has not enough time to recover the tangential direction. It recovers it only somewhat beyond the ideal perihelion point. In this way the perihelion advances a little bit in the prograde sense in each orbital roundtrip.

VII-8. Absence of effects of the solar gravitational field on the GPS clocks.

The velocity of clocks, moving with earth round the sun, with respect to the HQS is:

$$
\begin{aligned}
\vec{v} & =\vec{V}(r)+\vec{V}_{i m p} \\
& \cong(\gamma M / r)^{1 / 2} \vec{e}_{\phi}-(\gamma M / r)^{1 / 2} \vec{e}_{\phi} \approx 0
\end{aligned}
$$

Such clocks are stationary with respect to the HQS in the solar Keplerian velocity field. As the gravitational time dilation is caused by velocity of the clocks with respect to the HQS, these clocks may show no gravitational time dilation as indeed observed for the GPS clocks that move with earth round the sun. All clocks orbiting in circular equatorial orbits round an astronomical body, which normally orbits itself in a circular equatorial orbit round a larger body, are naturally synchronous and all show closely the same proper time throughout the universe.

VII-9. Effects of the velocity field of the HQS Eq.(3) on clocks moving in non-equatorial circular orbits.

Clocks moving along polar orbits have, besides the implicit velocity along $-\phi$, given by Eq.(4), the velocity along the polar orbit. Hence, their velocity $v$ with respect to the HQS is $v=\sqrt{2} V$ and the time dilation is two times larger than that of identical clocks resting at the same altitude. Moreover, the velocity $v$ with respect to the QS of clocks in retrograde circular equatorial orbits is $v=2 \mathrm{~V}$ and their time dilation is four times larger than that of an identical clock resting in the gravitational field at the same altitude. In particular, the GPS satellites move at $2.02 \cdot 10^{4} \mathrm{~km}$ of altitude, along circular orbits inclined 55 degrees with respect to the earth's equator and hence their clocks have velocity components with respect to HQS $v_{o}(1-\cos \alpha)$ along $-\phi$ and $v_{o} \sin \alpha$ along $\pm \theta$, where $v_{o}=3.87 \mathrm{~km} / \mathrm{sec}$ and $\alpha$ is the angle of the orbital velocity $v_{o}$ with respect to the equator or parallels. The effective velocity is $v_{0}[2(1-\cos \alpha)]^{1 / 2}$ and the estimated average velocity of the GPS satellites over the entire orbit is $\sim 0.8 v_{0}$. Considering in addition the velocity of $0.22 \mathrm{~km} / \mathrm{sec}$ of the $C s$ atoms in the atomic clocks, this makes $3.30 \mathrm{~km} / \mathrm{sec}$ with respect to the HQS. Analogously, for the earth based stations we find $7.20 \mathrm{~km} / \mathrm{sec}$ with respect to the HQS. Using these values $\left(7.2^{2} / c^{2}-3.3^{2} / c^{2}\right)$, we find that the rate of the GPS clocks must be slowed by $4.5 \times 10^{-10} \mathrm{sec} / \mathrm{sec}$ before launch in order to these clocks to run approximately synchronous with identical clocks resting on ground. This is closely the value by which NASA slows the rate of the GPS clocks before launch.

VII-10. The astronomical motions closely track the motion of the HQS throughout the universe.

The null results of the light anisotropy experiments, due to the orbital and cosmic motion of earth and more emphatically the absence of the gravitational time dilation on the GPS clocks, due to the solar gravitational field, proves that the planet earth is stationary with respect to the local HQS that rules the propagation of light. From the HQS point of view, the planetary orbits lie all closely within the equatorial plane of the solar Keplerian velocity field of the HQS and the orbit of the solar system lies within the galactic disk, which is the equatorial plane of the galactic velocity field of the HQS. Hence, the planets as well as the solar system are commoving with the HQS. All these bodies minimize their velocity with respect to the HQS. This minimization visibly is the result of the expansion of the universe and the velocity averaging down during agglomeration into large matter bodies. The expansion of the universe must have stretched the wavelengths $(\lambda)$ of the particles analogously as it has stretched that of the 
photons, as is well known from the cosmic microwave background radiation.

VII-11. The Null Light Anisotropy and the Global Dynamics of the Universe.

Besides showing that earth is stationary with respect to the HQS, despite its orbital motion in the solar system and the motion of the solar system within the galaxy, the null light anisotropy also shows that the recession between the galaxies, too gives no rise to motion of earth with respect to the HQS. This becomes possible only if the expansion of the universe is expansion of the HQS itself. The energy, driving the accelerated expansion of the universe is estimated to involve mass-energy sufficient to create 14 universes like ours. There also is now consensus that this dark energy acts only by gravity and is distributed homogeneously all over the universe, so that its density is low. The source of such huge amount of energy cannot be provided by any interaction mechanism between the ordinary matter in the universe and hence must be searched in the very physics creating the universe. This idea is not at all naï. During condensation, the Higgs bosons liberate hundreds of $\mathrm{GeV}$ per Higgs boson, which signals very strong quantum correlation between them. Moreover, the energy of the HQS is not usual mass-energy. It is energy of space itself and is intrinsically distributed homogeneously throughout space. Its only relationship with ordinary massenergy is ruling the inertial motion and hence dynamics of the HQS can only generate gravitational effects. Initially, the density $\rho=\phi^{*} \phi$ of the Higgs condensate (HC) certainly was lower than the equilibrium density $n / m$ (see Equation (1)). The volume of the $\mathrm{HC}$ is not limited to a definite volume, likewise the superconducting condensate (SCC). Hence, in this scenario, the $\mathrm{HC}$ could lower its energy by volumetric contraction, possibly with formation of droplets, analogously as fluctuations of the SCC during the superconducting transition. However, at a given stage, $\rho$ must have achieved the equilibrium value, after which decelerating contraction may have driven $\rho$ to a maximum, on from which an accelerated expansion may have initiated. This sequence must have triggered a Higgs mode of the HQS. Actually, the HC still must be struggling against the perturbations, represented by the usual mass-energy, toward the minimum of energy. However, the HQS certainly is highly conservative, analogously as superfluids and the SCC, so that this Higgs mode may be persistent.

\section{Conclusions}

Several trustworthy experimental observations, recently achieved with the help of the tightly synchronized clocks in orbit, suggest a completely new scenario concerning the nature of space and of gravitation. This new scenario also finds support in the Higgs theory, underlying the Standard Elementary Particle Model. The new conception links together the central idea of Einstein's GR, according to which the gravitational pull is an inertial pull and the idea of the Higgs theory, according to which the Higgs Quantum Space (HQS) rules the inertial motion of matter and the propagation of light. This is the feasible unification of GR and of the Quantum Theory. The only way to the HQS generate the gravitational effects equivalent to the inertial effects, without running into conflict with experimental observations, is by moving round the astronomical bodies according to a Keplerian velocity field. Inertial motion of matter and propagation of light in this Keplerian velocity field appropriately gives rise to the observed gravitational dynamics on earth, in the solar system and within the galaxies. In this context, the gravitational fields are a residual macroscopic manifestation of the Higgs mechanism confining and compressing matter into compact bodies thereby lowering the energy of the Higgs condensate in the Higgs potential well (Fig.1).

\section{REFERENCES}

[1] Y. Aharonov and D. Bohm, Phys Rev. 115, 485, (1959).

[2] P.W. Anderson, Phys. Rev. 130, 439 (1963).

[3] Lance Dixon, slac.stanford.edu/pubs/beamline/26/1/261-dixon.pdf (1996).

[4] Y. Nambu, Physical Review 117, 648, (1960).

[5] J. Goldstone, A. Salam, S. Weinberg, Phys. Rev., 127, 965 (1962).

[6] P. W. Higgs, Physical Review Letters, 13, 508 (1964).

[7] F. Englert and R. Brout, Phys. Rev. Lett. 13, 321, (1964).

[8] T. W. B. Kibble, Scholarpedia, 4(1):8741, (2009).

[9] R.R. Hatch, Physics Essays 20, 83 (2007).

[10] R.R. Hatch, GPS Solutions 8, 67 (2004).

[11] R.R. Hatch, Foundations of Physics, 34, 1725 (2004).

[12] J. Schaf, Recent Progress in Space Technology, 4, 44 (2014).

[13] J. Schaf, Journal of Modern Physics, 5, 407 (2014).

[14] J. Schaf, Journal of Modern Physics, 5, 1617 (2014).

[15] W. Meissner and R. Ochsenfeld, Naturwissenschaften 21 (44): 787ÃćâĆňâĂIJ788 (1933).

[16] H. E. Ives and G.R. Stilwell, J. Opt. Soc. Amer., 28, 215, (1938).

[17] D. C. Miller, Review of Modern Physics, 5, 203 (1933).

[18] R.V. Pound and J.L. Snider, Phys. Rev. B, 140, B788 (1965).

[19] Neil Ashby, Private communication, Nov. 52012.

[20] H. Bailey, K. Borer, F. Combley, H. Drumm, F. Krienen, Nature 268, 5618 (1977).

[21] J. Schaf, Journal of Modern Physics, 6, 224, (2015).

[22] J. Schaf, Journal of Modern Physics, 3, 714, (2012).

[23] R. E. Salant, The J. of the Acoust. Soc. of Am. 46, 1153 (1969).

[24] J.W. Brault, Bull. of the Am. Phys. Soc. 8, 28 (1963).

[25] I.I. Shapiro et ali, Phys. Rev. Lett. 26, 1132, (1971). 